\title{
Branched-Chain Amino Acid Therapy for Spinocerebellar Degeneration: A Pilot Clinical Crossover Trial
}

\author{
Nozomi Mori, Yoshiki Adachi, Takao Takeshima, Yoshihiro Kashiwaya, \\ Akitsugu OKada and Kenji NaKashima
}

\begin{abstract}
Object: The potential effects of branched-chain amino acids (BCAAs) on spinocerebellar degeneration (SCD) were explored in eleven patients. Methods: The patients received $200 \mathrm{ml}$ of BCAA-rich solution, $2 \mathrm{mg}$ of thyrotropin-releasing hormone (TRH; protirelin), or a placebo daily for 7 days each in a random order. An SCD score was used to quantify the severity of symptoms. Patients: Eleven patients with SCD ( 7 male, 4 female; mean age 60 \pm 11 ; mean disease duration 5.5 years) participated in this study. Results: The mean SCD score of the eleven patients improved significantly by the BCAA treatment compared with the baseline. The conditions of five of the eleven patients (45\%) were clearly improved by the BCAA treatment. All of the responders manifested predominantly cerebellar symptoms, but no prominent parkinsonian symptoms. Two patients with marked rigidity and akinesia did not respond to the treatment. Conclusion: We concluded that BCAAs do have a beneficial effect on functional improvement in patients with SCD, and that further large scale studies are needed.
\end{abstract}

(Internal Medicine 38: 401-406, 1999)

Key words: ataxia, spinocerebellar degeneration (SCD) score, posturography method, thyrotropinreleasing hormone tartrate (TRHT)

\section{Introduction}

Degeneration and loss of cerebellar neurons including Purkinje cells and granule cells are the main cause of spinocerebellar degeneration (SCD). Many studies have been performed to design a treatment protocol for SCD (1-3), but few satisfactory therapies have been established. Thyrotropin-releasing hormone (TRH) has had a central role in SCD treatments, and several studies of this agent have been conducted $(1,3,4)$. However, its effectiveness varies with the type or severity of disease, and some investigators report that TRH has no significant effect compared with a placebo (4).

We have been searching for a novel therapy to slow or halt cerebellar degeneration and to recover motor function. Although excess glutamate has excitatory neurotoxicity in the extracellular fluid, glutamate is an important neurotransmitter between cerebellar granule cells and Purkinje cells. We have speculated that stimulation of intracellular glutamate metabolism may improve the neurotransmission of cerebellar neurons. In other words, administration of amino acids that supply the amino group in glutamate synthesis may improve motor function and inhibit neuronal degeneration. Branched-chain amino acids
(BCAAs), as described by Yudkoff, are the most suitable amino acids for this purpose (5). We evaluated the potential neurotrophic effects of BCAAs using rat primary cerebellar cultures, and observed that BCAA treatment significantly increases neuronal survival in vitro (6). This result indicated the potential beneficial effects of BCAAs for SCD. Thus, we carried out a single-blind triple-crossover trial of BCAA therapy in patients with SCD.

\section{Subjects and Methods}

Eleven patients participated in this study (Table 1). They were admitted to the neurological ward of Tottori University Hospital between January and October 1997. The patient group consisted of seven men and four women. The mean age was $60 \pm 11$ (SD) at admission, and the mean duration of disease was 5.5 years. One patient had genetically proven spinocerebellar atrophy (SCA) type 1 and another had SCA type 6. The remaining cases were sporadic (seven patients had olivopontocerebellar atrophy (OPCA) and two had late cerebellar cortical atrophy (LCCA)). The severity of illness was classified according to the ordinal scale designed by Hirayama

From Division of Neurology, Institute of Neurological Sciences, Tottori University, Faculty of Medicine, Yonago

Received for publication June 1, 1998; Accepted for publication December 7

Reprint requests should be addressed to Dr. Nozomi Mori, Division of Neurology, Institute of Neurological Sciences, Tottori University, Faculty of Medicine, 36-1 Nishimachi, Yonago, Tottori 683-8504 
Mori et al

Table 1. Summary of the Study Population of Spinocerebellar Degeneration Patients

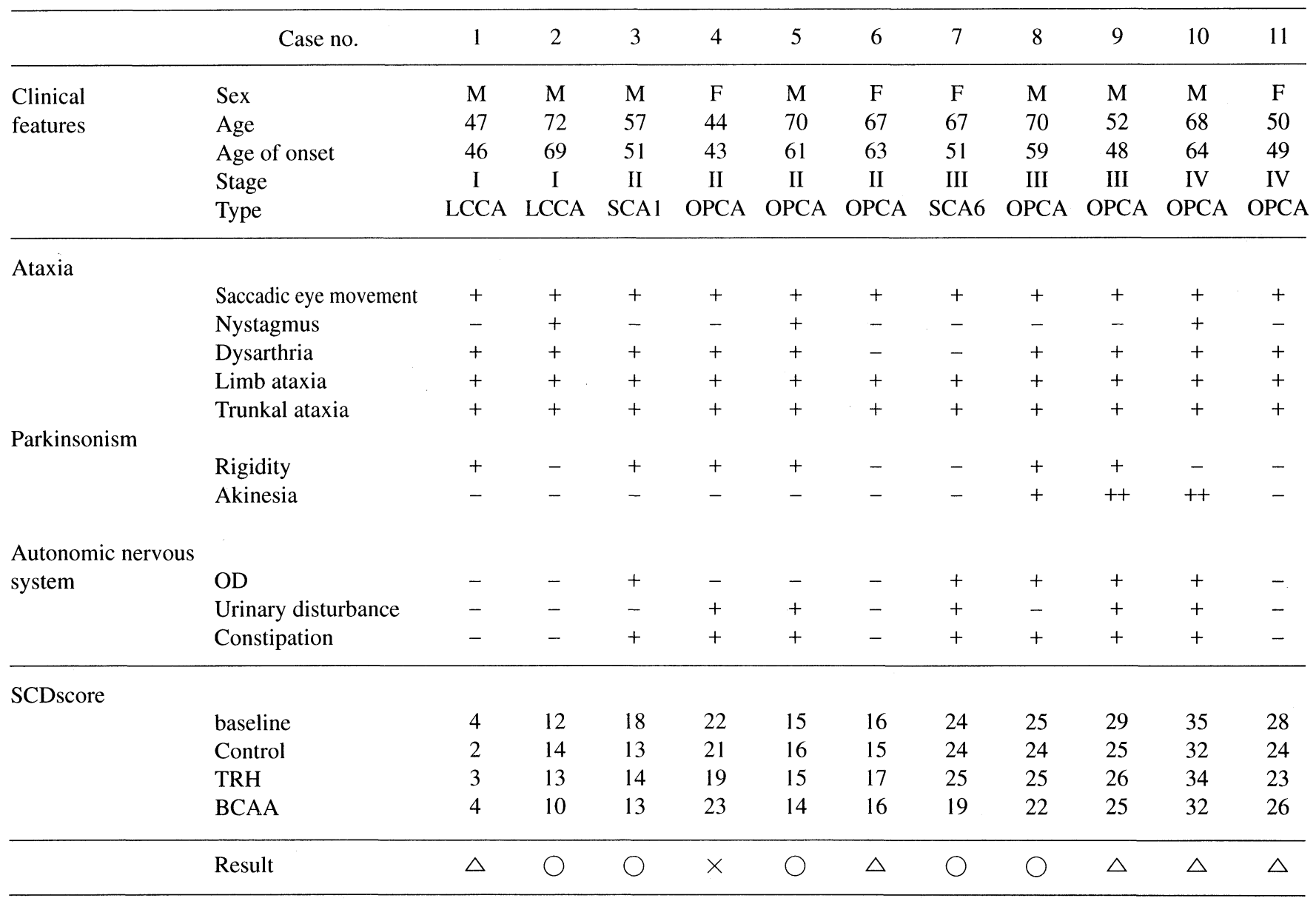

LCCA: late cerebellar cortical atrophy, OPCA: olivopontocerebellar atrophy, SCA 1: spinocerebellar atrophy type 1, SCA 6: spinocerebellar atrophy type 6, OD: orthostatic dysregulation, TRH: thyrotropin-releasing hormone, BCAA: branched-chain amino acid. $\bigcirc:$ effective, $\triangle$ : no change, $x$ : worsened.

et al; stage 1: independent gait; stage 2: gait with occasional assistance by an attendant or with aids; stage 3: gait with constant assistance by an attendant; stage 4: independent ambulation with a wheelchair; stage 5: bed-ridden (7). Two patients were at stage 1 , four at stage 2 , three at stage 3 , and two at stage 4. Informed consent was obtained from all of the patients after the nature of this trial was fully explained. The patients received $200 \mathrm{ml}$ of BCAA-rich solution [Aminoleban ${ }^{\circledR}$ (Otsuka Pharmaceutical Co., Ltd., Tokyo)], 2 mg of TRH (protirelin) dissolved in $200 \mathrm{ml}$ of solita $\mathrm{T}^{\circledR}$ or a placebo (200 $\mathrm{ml}$ of solita $\mathrm{T}^{\circledR}$ ) daily in the morning for 7 days each, in random order.

Aminoleban ${ }^{\circledR}$ is widely used for the treatment of hepatic encephalopathy (Table 2). The patients were masked with respect to which treatment they were receiving at a given time. SCD scoring was created, and has been utilized, in our ward for the clinical assessment of SCD based on the Ataxia Evaluation Manual in a multi-center, placebo-controlled double-blind study of thyrotropin releasing hormone tartrate (TRH-T) (1). Patients were scored before commencing (baseline) and fol-
Table 2. Contents of Aminoleban ${ }^{\circledR}$

\begin{tabular}{ll}
\hline Contents & $(\mathrm{g} / 1.0 \mathrm{~L})$ \\
L-Threonine & 4.5 \\
L-Serine & 5.0 \\
L-Proline & 8.0 \\
L-Cysteine hydrochloride & $0.4(0.3 \mathrm{~g}$ as L-Cysteine $)$ \\
Aminoacetic acid & 9.0 \\
L-Alanine & 7.5 \\
L-Valine (Val) & 8.4 \\
L-Methionine & 1.0 \\
L-Isoleucine (Ile) & 9.0 \\
L-Leucine (Leu) & 11.0 \\
L-Phenylalanine (Phe) & 1.0 \\
L-Tryptophan (Ryr) & 0.7 \\
Lysine hydrochloride & $7.6(6.1 \mathrm{~g}$ as L-Lysine) \\
L-Histidine hydrochloride & $3.2(2.4 \mathrm{~g}$ as L-Histidine) \\
Arginine hydrochloride & $7.3(6.0 \mathrm{~g}$ as L-Arginine) \\
\hline
\end{tabular}

BCAA (Val+Leu+Ile): 2.84 (W/V\%). Fischer ratio: 37.05 (Val+Leu+Ile)/(Phe+Tyr) (mo/mol). 
lowing treatment in order to quantify the severity of, and changes in, clinical symptoms. For all scale items, scoring ranged from 0 (normal) to 45 (poorest performance). The posturography method was used to document and quantify truncal ataxia, except for the wheelchair-bound patients. The parameters of trace length per environ area of sway (L/E area) and root mean square area (RMS area) were used for the evaluation with a static posturographic platform (stabilometry) Gravicorder $^{\circledR}$ (Anima, Japan). Clinical symptoms, SCD scores, and posturography measurements were evaluated after each seven-day treatment. Serum and cerebrospinal fluid (CSF) amino acids were analyzed at the baseline and at the end of the BCAA treatment phase.

\section{Results}

\section{Analysis of 11 cases}

Five of the eleven patients showed definite subjective and objective improvement after BCAA treatment (effective ratio $45.5 \%)$. Five patients showed no remarkable change $(45.5 \%)$, and the condition of one patient worsened (9.0\%). The responders were all at the mild or moderate (1 through 3 ) stages. The moderate cases showed definite improvement after treatment with BCAA, while the milder or more severe cases had no similar improvement (Fig. 1). For the total patient series, the

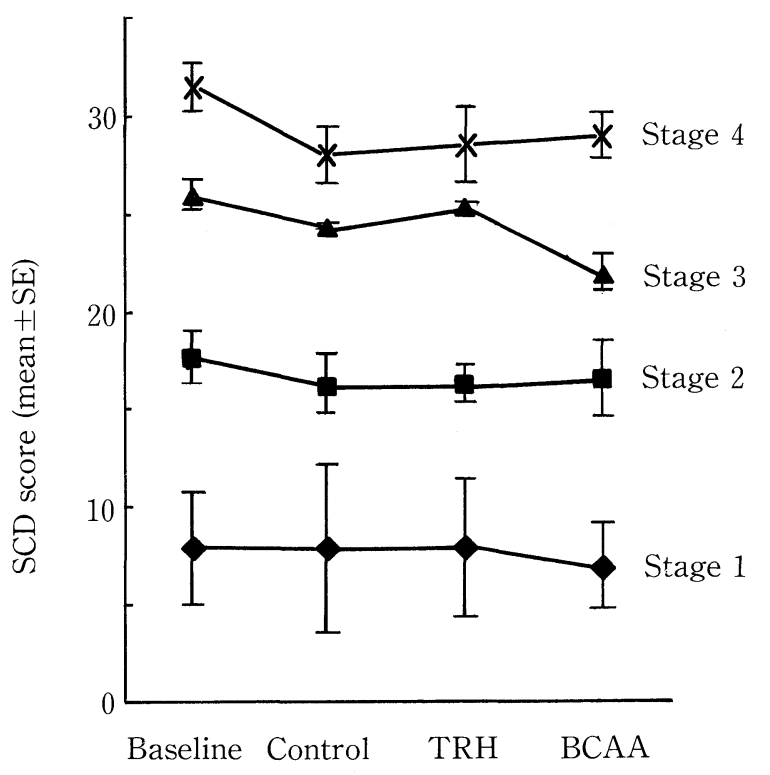

Figure 1. SCD score changes in patients at each stage of disease severity. The changes in the SCD score with each treatment are shown in the patient groups at each stage of the disease. Eleven patients received $200 \mathrm{ml}$ of BCAA-rich solution, $2 \mathrm{mg}$ of TRH (protirelin) or a placebo, as a control, daily in the morning for 7 days each, in a random order. SCD scores were evaluated at the end of each phase. Only the stage 3 patients (Hirayama's classification) showed a sensitive response to BCAA treatment; but patients at other stages (more mild or more severe) showed no particular tendency. Lower scores indicate better performance. Error bar: standard error. mean SCD score at the baseline was $20.7 \pm 8.8$ (mean \pm SE), which improved significantly to $18.6 \pm 8.1$ following BCAA treatment (Fig. 2: analysis of variance (ANOVA) $\mathrm{p}<0.02$, post hoc $\mathrm{p}<0.05)$. Differences among the control, TRH, and BCAA treatments were not significant.

In the nine items of SCD score, only hand-writing, that is, drawing spirals and tracing mazes, showed obvious improvement after BCAA treatment (Fig. 3: paired Wilcoxon test $\mathrm{p}<0.05)$. Neither posturography measurements nor amino acid content of serum or CSF showed significant change over the study duration (Figs. 4 and 5). The concentration of CSF glutamate was not detectable in four patients and traces were observed in two others.

Five patients, four men and one woman (mean age; 67.2 \pm 6.0 years), responded to BCAA therapy. The mean period of the disease for these patients was $9.0 \pm 5.0$ years. Their clinical types were variable; two cases of hereditary cerebellar ataxia (SCA 1, SCA 6), two OPCA and one LCCA. All manifested predominantly cerebellar symptoms, but no prominent symptoms of parkinsonism. The SCD scores of the responders were $18.8 \pm 5.6$ at the baseline, $18.2 \pm 5.4$ after control, $18.4 \pm 6.0$ after TRH, and 15.6 \pm 4.8 after BCAA treatment (Fig. 6). Significant changes were observed between BCAA and baseline, BCAA and control, and BCAA and TRH (ANOVA $<0.02$, post hoc

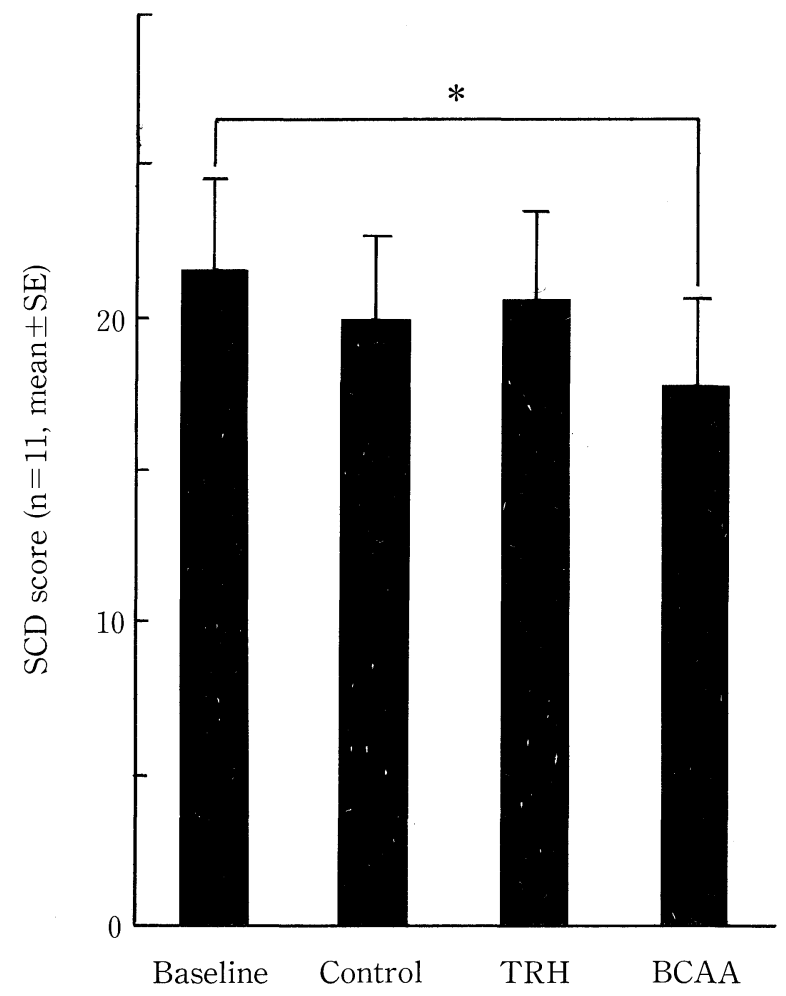

Figure 2. Changes of SCD scores after BCAA or TRH treatments. The mean SCD scores of all patients are illustrated. The scores improved significantly after BCAA treatment compared with the baseline (*: ANOVA $p<0.02$, post hoc $p<0.05$ ). The differences among the placebo (control), TRH, and BCAA treatments were not significant. Error bar: standard error. 


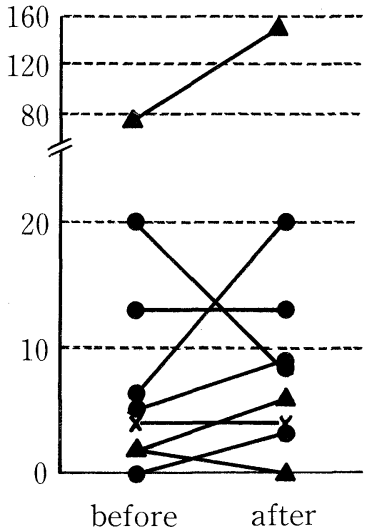

Standing

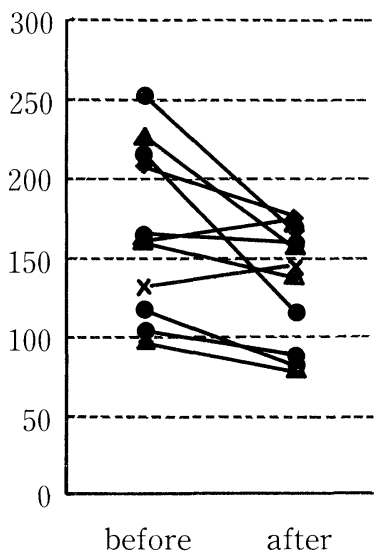

Drawing

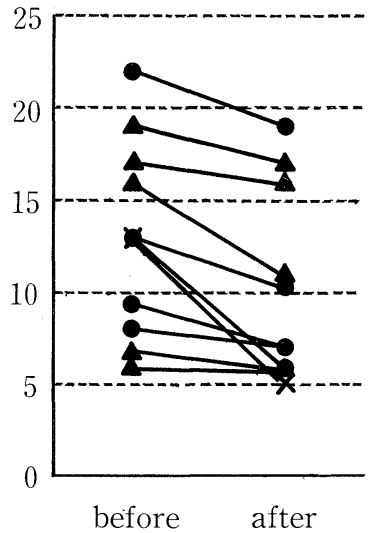

Speaking

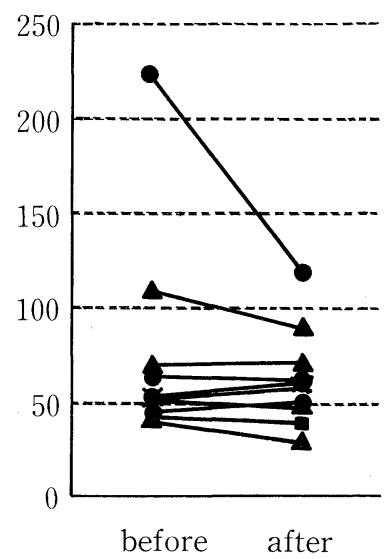

Heel-shin

Figure 3. The changes of four components of the SCD score after BCAA treatment ( $n=11)$. The figure illustrates four out of the nine components comprising the SCD score. Closed circles and triangles indicate the responders $(n=4)$, and the non-responders $(n=2)$ respectively, and an $X$ represents the patient whose condition worsened. After BCAA treatment, the time standing on one foot (right+left) improved in seven patients, the time to repeat a sentence three times ('Ruri mo Hari mo Teraseba Hikaru' in Japanese) improved in ten, drawing spirals on predrawn patterns and tracing mazes in nine, and heel-shin test in six patients. Of the nine components of the SCD score, only handwriting, that is, drawing spirals and tracing mazes, showed obvious improvement after BCAA treatment (paired Wilcoxon test $\mathbf{p}<\mathbf{0 . 0 5}$ ). Lower scores indicate better performance in all items except standing.

$\mathrm{p}<0.05)$, but not between baseline, control, and TRH scores (Fig. 6).

\section{A case report}

Here we present a patient with remarkable effects after BCAA therapy. A woman, aged 66, developed gait disturbance and dysarthria at age 48, and was diagnosed as having LCCA at age 56. She had no family history of ataxia and there was nothing particularly noticeable in her medical or life history. Neurologically, she showed scanning and explosive speech,

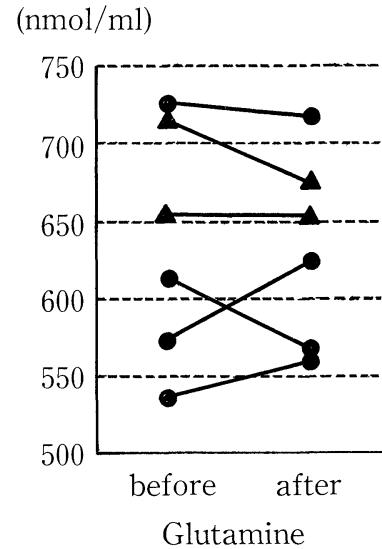

$(\mathrm{nmol} / \mathrm{ml})$

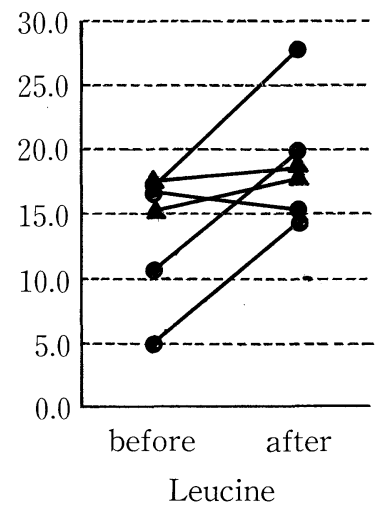

$(\mathrm{nmol} / \mathrm{ml})$

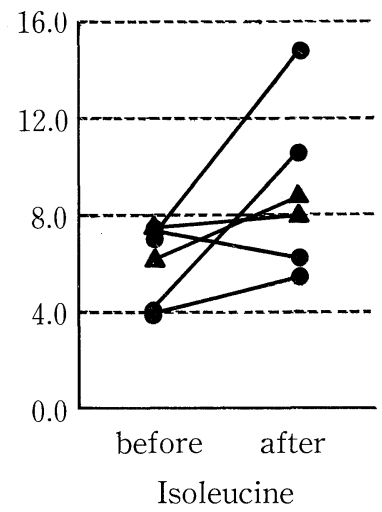

Figure 4. Changes in the content of selected amino acids in cerebrospinal fluid after BCAA treatment. Lumbar puncture was performed in six patients at the baseline and the end of BCAA treatment. Closed circles and triangles indicate the responders $(n=4)$, and the non-responders $(n=2)$ respectively. After administration of BCAA, concentrations of valine, leucine, and isoleucine seemed to become elevated, but there were no significant differences between before and after the treatment.

blurred vision with gazing nystagmus, cerebellar ataxia including limb ataxia, dysdiadochokinesis, dysmetria, intentional tremor of fingers, and ataxic gait. Deep tendon reflexes were normal and no rigidity was observed. The CAG repeat of $\alpha_{1 A^{-}}$ subunit-voltage-dependent calcium channel was extended to 22 , and the patient was diagnosed as having SCA6. She was classified as stage 3 . Her SCD scores were 24 at baseline, 24 after control, 25 after TRH treatment, and 19 after BCAA treatment. BCAA administration markedly improved her SCD score. She was able to walk more easily after the BCAA feedings, and her ataxic symptoms significantly improved both subjectively and objectively, especially regarding discrete movements such as speed of handwriting and tapping. The time required to trace pre-drawn spirals and mazes improved by 116 seconds after BCAA treatment, compared with 216 seconds at baseline. Her posturography also markedly changed: the RMS area (mean $3.2 \pm 1.4$ ) improved from $5.06 \mathrm{~cm}^{2}$ to $3.52 \mathrm{~cm}^{2}$, and the $\mathrm{L} / \mathrm{E}$ area (mean $23.3 \pm 7.5$ ) improved from $13.21 \mathrm{~cm}^{-1}$ to $20.62 \mathrm{~cm}^{-1}$ 


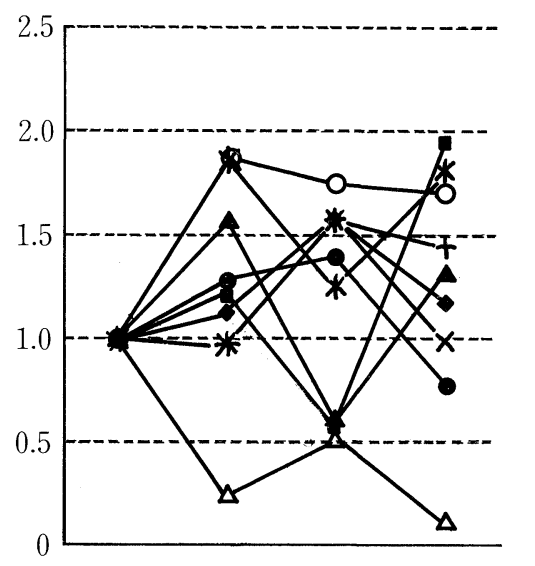

$\multimap$ Case no. 1

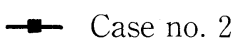

$\boldsymbol{-}$ - Case no. 3

$-x-$ Case no. 4

-*- Case no. 5

$\rightarrow$ Case no. 6

-t- Case no. 7

-0- Case no. 8

$\neg \quad$ Case no. 9

Baseline Control TRH BCAA RMS. Area

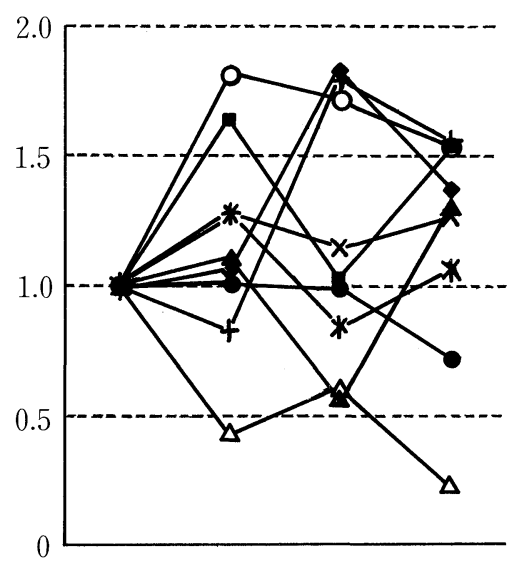

Baseline Control TRH BCAA L/E Area

Figure 5. The changes of posturogram after each treatment. Nine patients underwent posturography at the baseline and after each treatment to document and quantify truncal ataxia, while the other two patients did not because they could not stand without support for 60 seconds. The parameters of trace length per environ area of sway (L/E area) and root mean square areas (RMS area) were evaluated with a static posturographic platform (stabilometry) Gravicorder ${ }^{\circledR}$ (Anima, Japan). The improvement rates of the two parameters compared with the baseline are illustrated. The parameters did not show any significant changes.

after BCAA treatment. No adverse side effects were observed throughout the treatment.

\section{Discussion}

Amino acids that supply the amino group in glutamate synthesis in the central nervous system must satisfy the following criteria: (a) the donor amino acid must transport rapidly across the blood-brain barrier, (b) the donor itself must not be neuroactive, i.e., it must safely "travel" throughout the brain extracellullar fluid and not evoke depolarization, and (c) the

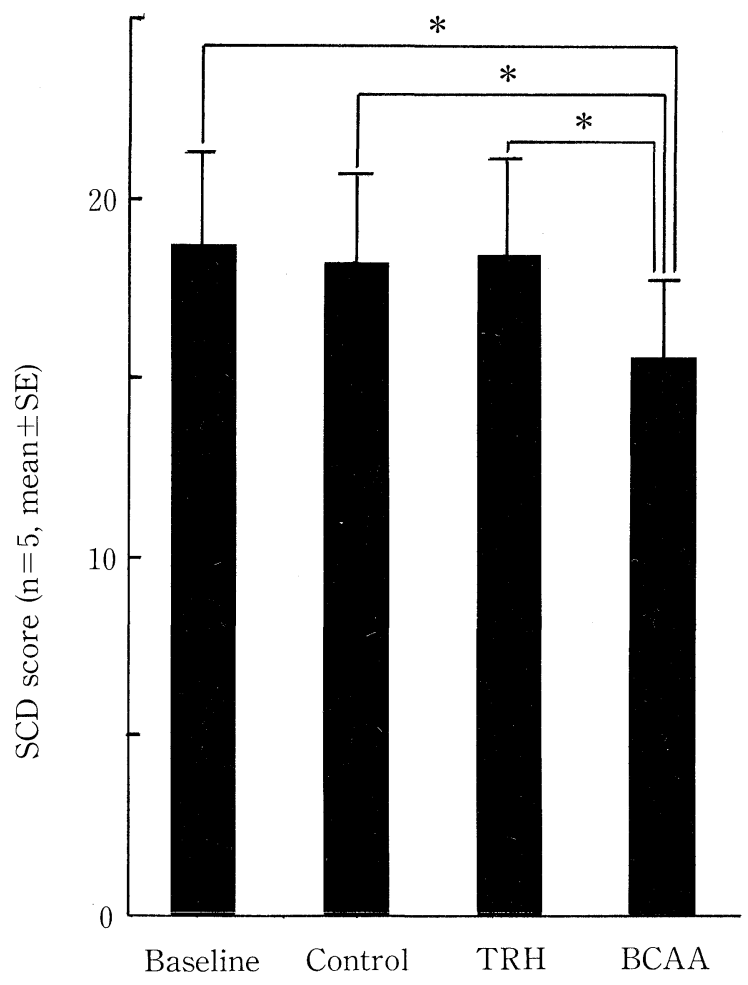

Figure 6. The changes of SCD scores in the responders with BCAA treatment. The mean SCD scores in the five responders with BCAA are shown. There were significant changes in the SCD score between BCAA and baseline, control, or TRH (*: ANOVA $<0.02$, post hoc $\mathbf{p}<0.05$ ). Error bar: standard error.

amino group of the donor must be readily transferred to $\alpha$ ketoglutarate, via transamination catalyzed by an enzyme abundant in the nervous system (5). Branched-chain amino acids can serve this metabolic and nutritional role. Thus, BCAA therapy may be expected to have some effect on the functional recovery of neurodegenerative diseases. BCAA administration significantly increased the survival of cultured cerebellar neurons (6), which suggests that BCAAs may act as a neuroprotective agent for cerebellar neurons. The use of BCAAs to buffer extracellular glutamate pools and as a treatment in patients with amyotrophic lateral sclerosis has been reported (8-11), however, we could find no such report on the effect of BCAAs on SCD.

For evaluation, we adopted the SCD score, established for clinical evaluations of the symptoms of SCD patients based on the ataxia evaluating manual of TRH-T in a multi-center, placebo-controlled double blind study (1). The International Cooperative Ataxia Rating Scale, which the Ataxia Neuropharmacology Committee of the World Federation of Neurology proposed for the pharmacological assessment of cerebellar ataxia, was not yet available at the commencement of this trial (12). In our trial, patients who showed improvement were at the mild or moderate stages. For this reason, it is likely that the severe-stage patients had few surviving Purkinje cells and could not, therefore, respond to the stimulated glutamate release: In 
addition, little response was evoked from TRH therapy, which has been reported to have a beneficial effect on SCD in many studies. This lack of response may be due in part to the fact that the TRH was administered only for one week; as its recommended regimen is for a two-week period $(1,3,4)$. While there was no significant difference between the placebo (control) and TRH or BCAA treatments, the BCAA therapy showed a significant improvement in SCD scores relative to baseline (Fig. 2). The responders showed predominant cerebellar symptoms but no prominent parkinsonism. The patients with marked rigidity and akinesia (Cases 9 and 10) did not respond to the treatment. Large neutral amino acids (LNAAs) are known to compete with levodopa for transport across the blood-brain barrier (13). The BCAAs, valine, leucine and isoleucine, belong to this category of amino acids. Therefore, patients who respond to levodopa therapy are likely to experience an exacerbation of parkinsonian symptoms due to elevated plasma BCAA levels.

BCAAs, which are absorbed rapidly after injection, are distributed throughout the central nervous system and muscle, where they readily undergo metabolic changes. The BCAA concentration in the CSF does not necessarily reflect its importance in the central nervous system. Extracellular glutamate is also distributed at varying concentrations in the CSF (highest in the synapse cleft) and may be taken up rapidly. The CSF obtained by lumbar puncture may not accurately reflect the central nervous system BCAA concentration.

In this trial, we investigated the functional recovery of SCD patients with a short-term administration of BCAA, and we are thus not able to evaluate its effect on slowing the progression of the disease. From the results of this study, a long-term oral treatment with BCAA in patients with SCD seems prudent. For a short-term trial, an intravenous administration of BCAA may be a beneficial therapy for moderate stage patients (Stages 2 and 3) with the cerebellar-predominant form of SCD.

In conclusion, the clinical symptoms and SCD scores were significantly improved by BCAA treatment compared with baseline. Five of eleven patients with SCD (45.4\%) responded to the BCAA treatment. BCAA is a promising agent for the treatment of patients with SCD, especially for their cerebellar symptoms. Large-scale and long term studies are necessary to further investigate the efficacy of BCAA treatment.

This work was supported by a grant-in-aid for the Research Committee on Ataxia of the Ministry of Welfare and Health, Japan.

\section{References}

1) Sobue I, Takayanagi T, Nakanishi T, et al. Controlled trial of thyrotropin releasing hormone tartrate in ataxia of spinocerebellar degenerations. $\mathbf{J}$ Neurol Sci 61: 235-248, 1983.

2) Wessel K, Langenberger K, Nitschke MF, Kömpf D. Double-blind crossover study with physostigmine in patients with degenerative cerebellar diseases. Arch Neurol 54: 397-400, 1997.

3) Waragai M, Ogawara K, Takaya Y, Hayashi M. Efficacy of TRH-T for spinocerebellar degeneration - the relation between clinical features and effect of TRH therapy. Rinsho Shinkeigaku (Clin Neurol) 37: 587-594, 1997 (Abstract in English).

4) Hattori T, Asahina M, Shinoto H, Arai K, Hirayama K. Effect of a single dose of intravenous thyrotropin releasing hormone on postural instability in patients with cerebellar degeneration: a double-blind cross over study. Annual report of the Research Committee of Ataxic Diseases, the Ministry Health and Welfare of Japan, 1996: 61-64 (Abstract in English).

5) Yudkoff M. Brain metabolism of branched-chain amino acids. Glia 21: 92-98, 1997.

6) Mori N, Adachi Y, Takeshima T, et al. A trial of amino acid therapy against spinocerebellar degeneration. Annual report of the Research Committee of Ataxic Diseases, the Ministry Health and Welfare of Japan, 1998: 4850 (in Japanese).

7) Hirayama K, Takayanagi T, Nakamura R, et al. Spinocerebellar degenerations in Japan: a nationwide epidemiological and clinical study. Acta Neurol Scand Suppl 153: 1-22, 1994.

8) Plaitakis A, Smith J, Mandeli J, Yahr MD. Pilot trial of branched-chain aminoacids in amyotrophic lateral sclerosis. Lancet 1: 1015-1018, 1988.

9) Testa D, Caraceni T, Fetoni V. Branched-chain amino acids in the treatment of amyotrophic lateral sclerosis. J Neurol 236: 445-447, 1989.

10) The Italian ALS Study Group. Branched-chain amino acids and amyotrophic lateral sclerosis: A treatment failure? Neurology 43: 2466-2470, 1993.

11) Tandan R, Bromberg MB, Forshew $D$, et al. A controlled trial of amino acid therapy in amyotrophic lateral sclerosis: I. Clinical, functional, and maximum isometric torque data. Neurology 47: 1220-1226, 1996.

12) Trouillas $P$, Takayanagi $T$, Hallett $M$, et al, and The Ataxia Neuropharmacology Committee of the World Federation of Neurology. International Cooperative Ataxia Rating Scale for pharmacological assessment of the cerebellar syndrome. J Neurol Sci 145: 205-211, 1997.

13) Pincus JH, Barry KM. Plasma levels of amino acids correlate with motor fluctuations in parkinsonism. Arch Neurol 44: 1006-1009, 1987. 